\title{
Evidence based medicine and ethics
}

\author{
Tony Hope Editorial Associate, Journal of Medical Ethics
}

\section{A new journal}

Evidence based medicine is an important modern movement. It has its evangelists; it has its enemies; and now it has the journal: Evidence Based Medicine. One can tell that a movement is important when its detractors heatedly maintain that it is nothing new. 'Evidence based medicine?' one chemist said to me, 'What other kind of medicine could there possibly be?' and a consultant physician said gruffly: 'We have always practised evidence based medicine'.

My wife, who is a general practitioner, recently referred a fifty-year-old woman who was on hormone replacement therapy (HRT) for surgery. On the day of the operation, after she had been admitted, the woman was sent home by the anaesthetist because of the HRT. My wife believed that there was no good evidence that HRT increased the risks from surgery. She spoke to the anaesthetist and was told that it was 'received wisdom' that HRT increased morbidity. No further reasons were given or thought necessary. The hospital had no guidelines or policy on this issue. Evidence based medicine has developed as a critical response to just this kind of behaviour. It emphasises the point that it is not the authority of the doctor that justifies a particular clinical intervention, but the evidence for the intervention's effectiveness.

Davidoff and colleagues (1) identify five linked ideas as central to evidence based medicine. They write: 'Firstly, clinical decisions should be based on the best available scientific evidence; secondly, the clinical problem - rather than the habits of protocols - should determine the type of evidence to be sought; thirdly, identifying the best evidence means using epidemiological and biostatistical ways of thinking; fourthly, conclusions derived from identifying and critically appraising evidence are useful only if put into action in managing patients or making health care decisions; and, finally, performance should be constantly evaluated'.

Evidence based medicine recognises a hierarchy in the quality of evidence, with systematic reviews of randomised controlled trials at the top of this hierarchy. Retrospective studies are well down the list and clinical anecdotes are seen as providing little if any evidence for the value of an intervention.

\section{Autonomous authorisation}

Developments in medical ethics over the last twenty years have stressed the ethical importance of patient autonomy. Faden and Beauchamp (2) have coined the term 'autonomous authorisation' as a replacement for 'informed consent'. The patient, in their view, should authorise the doctor to carry out some management plan rather than simply consenting or not, to the doctor's proposals. Patient choice goes beyond consent and should involve the patient in the whole decision-making process. In order for such autonomous authorisation to be genuine both the patient and the doctor need access to good quality information. For this, if for no other reason, there is an ethical imperative for support of the fundamental tenets of evidence based medicine.

Evidence based medicine, however, can be seen as a tool, and tools can be used for good and for ill. It will be important to maintain an ethical critique of developments in evidence based medicine. There are several areas which need to be highlighted.

\section{Health care purchasing}

One of the major uses to which the ideas of evidence based medicine are being put is in the purchasing of health care. Purchasers are tending to use this information in order to buy those interventions for which there is good evidence that they are effective. That the purchasing of health care should take account of evidence for effectiveness must be a good thing, but I have three areas of ethical concern. The first is that evidence based medicine may be used as a tool to cut funding. The high levels of evidence which are espoused are available for only a small number of medical interventions. Evidence based medicine could provide those who wish to cut the costs of health care with an apparently value-free tool for excising large areas of health care expenditure.

My second area of concern is a more subtle variant of the first. The values of evidence based medicine could introduced a systematic bias into purchasing. This bias will be towards those treatments for which there is good evidence of effectiveness. There are at least two factors which influence whether such good 
quality evidence is available: the amount of effort which has been put into research; and the ease with which desired outcomes can be quantified. The enormous wealth within the pharmaceutical industry is likely to result in more research being carried out relating to drug treatments than to other forms of treatment. This could result in some drug treatments being recommended, and purchased, not because they are better than alternative, non-drug treatments, but because the evidence for effectiveness is better. And this evidence is better simply because more randomised controlled trials, for example, have been funded. The second factor is that some types of outcome are much easier to measure than others. For example the desired outcome for an intervention aimed at correcting an acute medical problem is likely to be easier to measure than the less clear-cut, longer term outcomes of interventions for people with chronic disease. Too rigorous a focus on evidence at the top of the 'evidence hierarchy' could lead to a systematic bias in purchasing which acts against the interests of those with chronic disabilities.

My third area of ethical concern is that a public health perspective may act against individual patient choice. Effectiveness is not the only factor relevant to patients. Too much reliance by purchasers on evidence based medicine may lead to treatments being unavailable because they do not represent the best buy'. The interests of patient choice must balance too ruthless a focus on the most effective treatments.

\section{Improving clinical practice}

Evidence based medicine is not only of interest to purchasers, but also of value in improving clinical practice. The move towards evidence based medicine has come mainly from the medical profession. In practice, therefore, this movement has tended to be doctor-centred rather than patient-centred. This doctor-centred bias has been clearly recognised within some sectors of the evidence based medicine movement. For example, there is a consumer section of the Cochrane Collaboration, a leading association of centres with a major interest in evidence based medicine. However, it remains broadly true that it is doctors who determine what questions should be addressed by research projects; doctors choose which questions should be the focus of systematic reviews; and doctors make the recommendations arising from those reviews. The objectivity and rationality which is part of the rhetoric of evidence based medicine tends to obscure the fact that values play an important role in the conclusions from evidence based medicine. For example, systematic review provides strong evidence that prophylactic antibiotics reduce the frequency of infection following caesarean section. The recommendation is that all women should be given prophylactic antibiotics (3). However, a patient representative looking through the data from the original studies was struck by the very different base rates for infection in different studies and in different communities (personal communication). The question which interested her was: what are the reasons for these huge differences? Her conclusion was not that all women should be given an antibiotic but that the reason should be sought as to why some units had a high infection rate. My point is not that one of these conclusions is the right one, but that each represents a perfectly rational response to the data. The conclusions from systematic reviews are affected by the reviewer's own values.

If evidence based medicine can benefit from ethical critique so too can ethics benefit from the values of evidence based medicine. There is, I believe, far too little interest shown within medical ethics circles, in relevant empirical matters or in the quality of evidence concerning those matters. Consider the following issue of confidentiality. A person with uncontrolled epilepsy does not inform the vehicle licensing authorities. The doctor knows that this person is still driving. The issue is whether the doctor should inform the licensing authorities. For those who tend to be persuaded by a consequentialist ethics, one of the most powerful arguments for maintaining confidentiality is the possible beneficial effect on patients' trust in doctors. Although people are at risk from this particular person's continued driving, more people would be at risk, the argument goes, if doctors breached confidentiality. The reason for this is that many people who have fits may not seek medical attention if they believe that their doctor will inform the licensing authority. The result: many more people driving around with uncontrolled epilepsy. This whole argument depends on empirical issues, but discussion in medical ethics rarely proceeds to serious consideration of these issues, or to an interest in how the relevant empirical information could be obtained.

The relationship between evidence based medicine and ethics is likely to be a fruitful source of philosophical analysis and empirical work.

\section{References}

(1) Davidoff F, Haynes B, Sackett D, Smith R. Evidence based medicine: a new journal to help doctors identify the information they need. British medical journal 1995; 310: 1085-1086.

(2) Faden R, Beauchamp T. A history and theory of informed consent. New York: Oxford University Press, 1986.

(3) Sheldon T. Not all health care is appropriate. In: Dunning M, Needham G, eds. But will it work doctor? The Consumer Health Information Consortium, 1994. 